\title{
Les protéines Rac : oncoprotéines et régulateurs du cytosquelette d'actine
}

Les protéines Rac 1 et Rac 2 sont des petites protéines $G$ appartenant à la famille Rho, sous-ensemble de la vaste superfamille des protéines Ras [1]; elles présentent des structures très voisines (92\% d'analogie), mais des spectres d'expression très différents: ubiquitaire pour Rac 1, restreint aux cellules myéloïdes et lymphoïdes pour Rac 2. Des travaux préalables avaient permis de les associer à deux types de fonctions: dans les phagocytes, Rac règle l'activité de la NADPH-oxydase, enzyme produisant les anions superoxyde indispensables à la fonction bactéricide; par ailleurs, l'équipe de Hall à Londres (GB) avait montré dans un modèle de fibroblaste de rat que Racl, en synergie avec une autre petite protéine $\mathrm{G}$, Rho, règle la réorganisation du cytosquelette d'actine induite en quelques minutes sous l'action de facteurs de croissance ou après micro-injection de Ras activé $[1,2]$.

Deux études récentes viennent d'enrichir et de modifier considérablement nos conceptions du rôle des protéines Rac. Dans la continuité de son travail antérieur, l'équipe londonienne vient de montrer que les modifications du cytosquelette d'actine qui sous-tendent les mouvements cellulaires sont contrôlées par l'activation concertée de trois petites protéines $\mathrm{G}$ de la famille Rho: Cdc42, Rac et Rho [3]. Les formes activées de ces petites protéines $\mathrm{G}$, microinjectées dans des fibroblastes Swiss3T3, induisent, via la polymérisation de l'actine sous-membranaire, la formation de trois types de structures membranaires distinctes. La protéine Cdc42, qui peut être activée par la bradykinine, provoque l'apparition de filopodia, excroissances très mobiles, capables d'adhérer réversiblement au support et pouvant $m / s n^{\circ} 7$, vol. 11 , juillel 95 atteindre 20 à $25 \mu \mathrm{m}$ de longueur. La voie $\mathrm{Rac}$, qui peut être activée par le PDGF (platelet-derived growth factor) et l'insuline, contrôle la formation de lamellipodia, sortes de "voiles" mobiles tendus entre deux filopodia voisins. L'activation de la voie Rho, induite en particulier par l'acide lysophosphatidique (LPA), entraîne la formation des câbles de tension (stress fibers). On savait déjà que la voie Rho contrôle également l'assemblage des plaques d'adhérence reliant les câbles d'actines aux intégrines. Il apparaît aujourd'hui que l'activation des voies Cdc42 et Rac induit aussi, au niveau de la membrane plasmique, des "complexes d'adhérence" plus petits que les plaques d'adhérence et spécifiques par leur aspect et leur distribution. Enfin, fait essentiel, les GTPases Cdc42, Rac et Rho sont organisées entre elles par une relation hiérarchique, l'activation de Cdc42 induisant de façon séquentielle celle de Rac puis celle de Rho. Ainsi, l'activation en cascade de Cdc42, Rac et Rho et l'enchaînement des événements qui en résultent (induction de filopodia, croissance de lamellipodia, adhérence et nouvel ancrage des câbles d'actine) fournit un modèle plausible des mécanismes qui permettent la progression du front d'une cellule en mouvement. Une étude également très récente provenant du groupe de McCormick (Onyx Pharmaceuticals, Richmond, CA, USA) vient de mettre en lumière deux résultats importants quant au rôle de Rac 1 dans l'oncogenèse [4]. D'une part, le gène racl activé par une mutation ponctuelle (VI 2) est lui-même transformant dans un modèle de fibroblaste de rat. Les lignées exprimant la protéine RaclV12 ont les propriétés de cellules transformées (prolifération accrue, perte partielle de l'inhibition de contact, moindre dépendance vis-à-vis du sérum, clonage en soft agar) et sont tumorigènes chez la souris athymique. D'autre part, le blocage de la voie Rac par un mutant trans-dominant négatif de racl (N17) inhibe la transformation par Ras de fibroblastes NIH3T3, ce qui implique que la voie Rac est essentielle pour la transformation par Ras. De plus, il existe une forte synergie pour la transformation de cellules NIH3T3 entre raclV12 et un gène raf activé. Pour induire la transformation, l'oncogène ras active donc à la fois la voie des MAP kinases et celle de Rac. Ces résultats placent donc les protéines Rac au cour d'un réseau complexe de voies de signalisation impliquées à la fois dans le contrôle par Ras de la prolifération cellulaire et dans la régulation, via le cytosquelette d'actine, des propriétés de forme, d'adhérence et de motilité cellulaires. La définition en termes moléculaires des voies de signalisation activatrices et effectrices des protéines Rac, et celle des relations entre Ras, Rac, Cdc42 et Rho, sont en cours. Il est certain que médecine/ sciences aura à revenir prochainement sur les résultats de ces travaux.

G.G.

1. Fort P, Vincent S. Transduction du signal mitogène, cytosquelette et petites protéines $\mathbf{G}$ : vers un réseau de protéines GAP? médecine/sciences 1993; 9: 59-65.

2. Ridley AJ, Paterson HF, Johnston CL, Diekmann D, Hall A. The small GTP-binding protein Rac regulates growth factor-induced membrane ruffling. Cell 1992; 70: 401-10.

3. Nobes CD, Hall A. Rho, Rac, and Cdc42 GTPases regulate the assembly of multimolecular focal complexes associated with actin stress fibers, lamellipodia, and filopodia. Cell 1995; 81: 53-62. 4. Qiu RG, Chen J, Kirn D, McCormick F, Symons M. An essential role for Rac in Ras transformation. Nature $1995 ; 374$ : 457-9. 